\title{
J-PARC COMMISSIONING RESULTS
}

\author{
Kazuo Hasegawa\# \\ JAERI, Tokai-mura, Naka-gun, Ibaraki-ken, 319-1195, Japan
}

\begin{abstract}
The J-PARC (Japan Proton Accelerator Research Complex) is a project to pursue frontier science and nuclear technology. It comprises a $400-\mathrm{MeV}$ linac, a 3$\mathrm{GeV}$ rapid-cycling synchrotron (RCS), a $50-\mathrm{GeV}$ main ring synchrotron (MR) and experimental facilities. The buildings and conventional facilities will be completed in succession in the Japanese Fiscal Year 2005 and 2006, when the installation of the accelerator components will be actually started at Tokai site. A peak current of $30 \mathrm{~mA}$ was accelerated up to $20 \mathrm{MeV}$ in the DTL beam commissioning at the KEK site and will be moved to Tokai. At the initial stage, the beam commissioning of the linac at an energy of $181 \mathrm{MeV}$ will be started in September, 2006, followed by the RCS and MR beam commissioning. The recent results of the developments of accelerator components, the present construction status and the commissioning schedule are presented.
\end{abstract}

\section{INTRODUCTION}

The High Intensity Proton Accelerator Project in Japan[1] is referred as "J-PARC Project", which stands for Japan Proton Accelerator Research Complex. This project is a joint one between the Japan Atomic Energy Research Institute (JAERI) and the High Energy Accelerator Research Organization (KEK).

The facility is under construction at the JAERI/Tokai site as shown in Fig. 1. An $\mathrm{H}^{-}$beam with a peak current of $50 \mathrm{~mA}$ and a pulse width of $500 \mu \mathrm{s}$ is accelerated up to $400-\mathrm{MeV}$ by a linac, and then injected to a $3-\mathrm{GeV}$ Rapid Cycling Synchrotron (RCS) with a repetition rate of 25 $\mathrm{Hz}$. The linac can be operated with a repetition rate of 50 $\mathrm{Hz}$, the remaining half of the beam will be used for the basic study of the Accelerator Driven Nuclear Waste Transmutation System (ADS) in the future.

The beam accelerated by the RCS with an average current of $333 \mu \mathrm{A}$ and a beam power of $1 \mathrm{MW}$ is fast extracted and transported to the Materials and Life Science Facility (MLF) most of the time. In the MLF, a muon and a neutron production targets are located in a series. About $10 \%$ of the beam is used for the muon production. Every 3.5 second, the beam is transported to a 50-GeV Main Ring Synchrotron (MR) and injected to it. The accelerated $50-\mathrm{GeV}, 0.75-\mathrm{MW}$ beam is slowly extracted to the Hadron Experimental Facility with a duration of $1.6 \mathrm{sec}$. Kaon rare decay experiments, hyper

\#hasegawa@linac.tokai.jaeri.go.jp

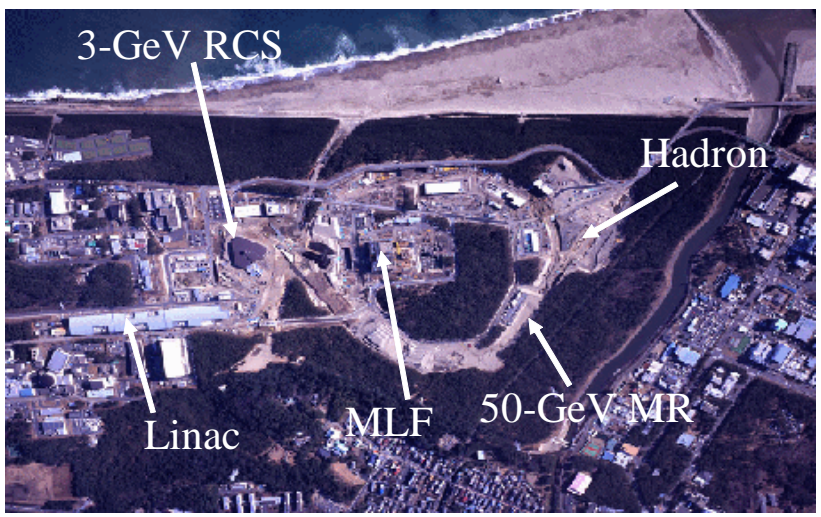

Figure 1: The J-PARC construction view at Tokai site (January, 2005).

nucleus experiments and so on will be conducted there. The beam is fast extracted to a neutrino production target. Produced neutrinos are sent to the SUPERKAMIOKANDE detector located $300 \mathrm{~km}$ west for long base line experiments.

The J-PARC project is divided into two phases. The construction of the Phase I started from April 2001 and will be completed by 2007 . The facilities in the Phase I comprises the linac, the RCS and the MR. The MLF is equipped with a full-power neutron production target. In the Phase I, however, the MR will be operated up to 40 $\mathrm{GeV}$, since the fly-wheel electric power system will be equipped in the Phase II. The Neutrino Facility was originally in the Phase II, but it was approved to construct from 2004, which implies that the Neutrino Facility is forwarded to the Phase I. The half of the Hadron Experimental Facility building is in the Phase I, and the remaining half will be constructed in the Phase II. The superconducting proton linac (SCL) from 400 to 600 $\mathrm{MeV}$ and the ADS experimental facilities are in the Phase II.

At the initial commissioning stage in the Phase I, the linac energy is decreased from $400 \mathrm{MeV}$ to $181 \mathrm{MeV}$ in order to compensate the budget overflow in the RCS and the linac. We decided to increase the beam aperture of the RCS in order to keep the sufficient margin for the space charge effort (the tune shift is 0.15 ). We also lengthen the RCS circumference by a factor of 10/9. The lowering of the linac energy will reduce the RCS beam power from $1 \mathrm{MW}$ to $0.6 \mathrm{MW}$. The present building can accommodate the $400 \mathrm{MeV}$ linac, and the energy recovery to $400 \mathrm{MeV}$ will be submitted to the funding agency after the completion of the initial stage of the Phase I. 
The funding to the linac and the RCS was delayed by one year from the original schedule. The schedule of the MR building was delayed by more than one year for the archaeological investigation of the ancient salt farms. The delay of the beam commissioning schedule, however, was managed to decrease to half a year. The schedule for the J-PARC Phase I is shown in Fig. 2.

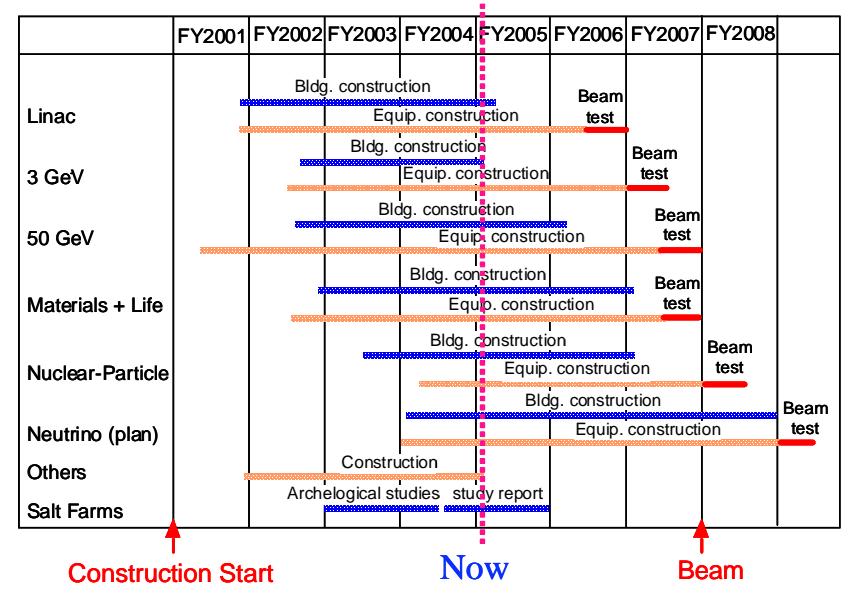

Figure 2: The schedule for the J-PARC Phase-I. The year shows the Japanese Fiscal Year which starts from April.

\section{FEATUES OF THE J-PARC ACCELERATOR}

The distinctive features of the J-PARC accelerator are arising from its multi-purpose concept; the realization of the high beam powers of MW in both several $\mathrm{GeV}$ and several tens of $\mathrm{GeV}$ region. The details of the accelerator design are reported in Ref.[2].

Since the $50-\mathrm{GeV}$ synchrotron requires several-GeV injection beams, the accelerator scheme is based on the RCS in contrast to the scheme of a full-energy linac and a storage ring option as the SNS project.

The choice of the high-power and high-energy RCS scheme makes us many issues to be solved. These are as follows,

1) The lower injection energy implies the higher space charge effect. A beam collimation system is prepared to localize the loss for blow-up beams. The large aperture magnets and powerful power supplies are also necessary. The injection scheme is hard to design for the large beam aperture. Leakage of the magnetic field is evaluating and the magnetic shield is carefully designing.

2) The fast accelerating system with high field gradient cavities is necessary. This was solved by the innovative development of the accelerating cavity loaded with magnetic alloy (MA).

3) To avoid eddy current effect arising from the fast changing magnetic fields, stranded conductor coils and ceramics vacuum ducts are needed.
4) Precise magnetic field tracking is necessary. Lower harmonic components and fast response can be obtained by using IGBT power supplies.

In addition to the challenging issues in the RCS itself, the linac is also required to provide the beam with low momentum spread $(\Delta \mathrm{p} / \mathrm{p}< \pm 0.1 \%)$ and with low transverse emittance $(<4 \pi \mathrm{mm}$.mrad). These values are necessary for the efficient painting and for the reduction of the beam loss in the RCS. In order to produce these high-quality beams, we designed and devised as follows,

1) We devised a pi-mode stabilizing loop to eliminate any effects of the deflecting field in the RFQ[3].

2) We decided to use electro-qudrupole magnets contained in the drift tubes in the DTL in order to keep the flexible knobs for the transverse tuning. The technology was newly developed for minimizing the size of the coils with water-cooling channels by using the electroforming method and wire cutting[4]. It brings to use frequency of $324 \mathrm{MHz}$ for the starting energy of $3 \mathrm{MeV}$ DTL.

3) In order to reduce beam losses in the RCS, a newly devised RF deflecting chopper is used[5].

4) A new structure, an SDTL (Separated-type DTL) [6], comprised of short tank containing 5 cells, is used. Tanks are so short that field stabilization is unnecessary and transverse focusing is provided between tanks.

5) An annular ring coupled structure (ACS) is chosen for its good axial symmetry, which may be important to minimize a halo formation.

6) In order to satisfy the requirement of the momentum spread, a debunching system is placed in the beam transport line between the linac and the RCS. Also, $\mathrm{H}^{-}$ particles outside of the transverse acceptance will be scraped by charge exchange into $\mathrm{H}^{+}$with scraper foils and will be dumped.

The RCS and the MR are designed on the basis of the lattices with low and negative momentum compaction factors, respectively, both of which imply no transition crossing during acceleration. The beam loss inherent to the transition crossing will be thus avoided.

\section{LINAC}

The linac comprised a volume-production type of $\mathrm{H}^{-i o n}$ source, a 3-MeV RFQ, a $50-\mathrm{MeV}$ DTL, a $190-\mathrm{MeV}$ SDTL, a 400-MeV ACS and a $600 \mathrm{MeV}$ SCL. Operation frequencies are $324 \mathrm{MHz}$ for the RFQ, DTL and SDTL and $972 \mathrm{MHz}$ for the ACS and SCL, respectively. As mentioned above, construction of the 190-400 MeV ACS linac is in the next step and it will be replaced by a beam transport at the first stage. The last two SDTL tanks are installed in the beam line as debuncher cavities and no acceleration is expected. Therefore, the injection energy to the RCS is $181 \mathrm{MeV}$. Most of the accelerator 
components were ordered by the end of JFY 2002 except for the ACS.

Fabrication of all the 3 DTL tanks and 32 SDTL tanks has been completed. So far, 8 SDTL tanks were high power tested over their design values. The DTL tanks were assembled and tuner positions and post coupler positions were adjusted until the uniform field distribution was realized within one percent accuracy (design value).

The RFQ linac used here was designed for the Japan Hadron Facility (JHF) project[7], which was original KEK's high intensity accelerator project. This RFQ would produce $0.6 \mathrm{MW}$ beams at $3 \mathrm{GeV}$ with a linac peak current of $30 \mathrm{~mA}$. The 50-mA RFQ linac for the final JPARC goal of $1 \mathrm{MW}$ is now under development.

The RF chopper at the Medium Energy Beam Transport (MEBT) was tested with the beam. The measured rise and falling times of the beam are $15 \mathrm{~ns}$, which were sufficiently fast for eliminating the beam loss as designed[8].

The DTL of the J-PARC linac consists of 3 tanks (50 $\mathrm{MeV}$ ) and beam commissioning up to the first tank (DTL$1,19.7 \mathrm{MeV}$ ) was carried out at KEK by September 2004[9]. The DTL-1 contains 76 acceleration cells and 77 electro-quadrupole magnets. The transmission efficiency through the DTL was 100\% within the current measurement errors of several percent. We use phaseamplitude scan method to adjust the phase and amplitude of RF cavities. Beam phases were measured with a Fast Current Transformer (FCT) located just after the DTL-1. Figure 3 shows the experimental results as well as the simulated phase-scan curves obtained with PARMILA code. The experimental results show good agreement with the simulation. These results demonstrate that the $1 \%, 1$ deg set-point accuracy will be achievable.

Figure 4 shows transverse emittances measured with emittance scanners located at downstream of the DTL-1.
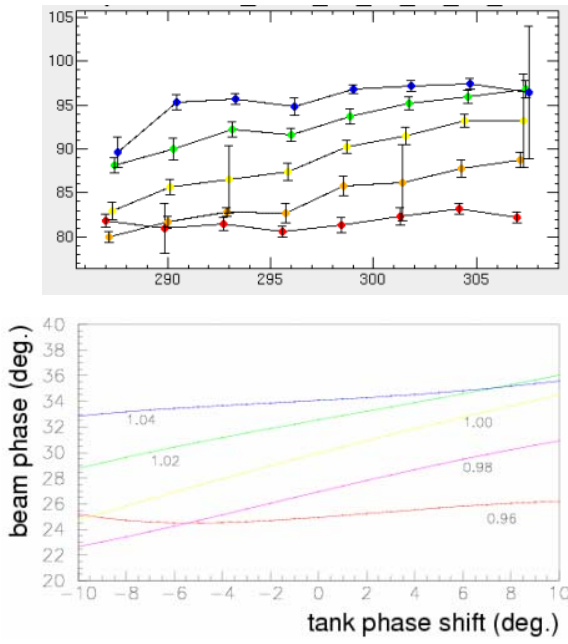

Figure 3: Phase-amplitude scan of the DTL-1. The experimental results (top) and PARMILA simulation results (bottom) for five different tank levels.
The measured normalized rms emittances at $25 \mathrm{~mA}$ were $0.3 \pi \mathrm{mm}$.mrd in both planes. Simulated values are 0.25 and $0.26 \pi \mathrm{mm} . \mathrm{mrad}$ in horizontal and vertical planes, respectively.

The accelerated peak current of $30 \mathrm{~mA}$ was already sufficient for the first goal of 0.6 MW at the extraction of the RCS to the MLF.

The linac building was accomplished at the end of this April and the machine installation has just started. In the Klystron gallery, installation of 19-inch racks as klystron stations and timing delivery system has been started. Klystrons will be conditioned from this July and will be installed. In the tunnel, we are testing an air ventilation and conditioning system and taking a couple of dry-up months. The RFQ, DTL and SDTL tanks will be moved from KEK and installed from this summer. We plan the beam test for the $181 \mathrm{MeV}$ linac in September, 2006. Since the RCS is under installation and testing at that time, the beam power will be limited by the allowed beam dump power and/or the radiation level at the boundary between the linac and the RCS.
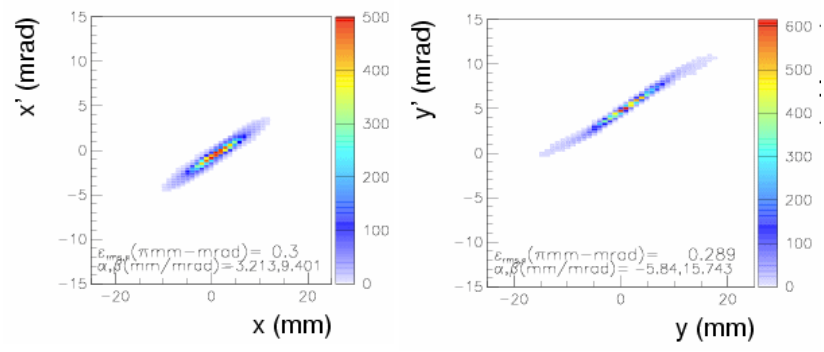

Figure 4: Measured horizontal (left) and vertical (right) emittances at the exit of the DTL-1.

\section{3-GEV RCS}

Most of the RCS components were ordered by the end of JFY 2004, including bending magnets (BMs), quadrupole magnets (QMs), magnet power supplies, RF sources, RF cavities, ceramics vacuum ducts, and all the kickers and their power supplies. The remaining some components for injection and extraction system will be contracted in JFY 2005.

The first BM and the QM were delivered and several kinds of measurements were carried out. The measured magnetic field results show good agreement with those of the design values. Temperature was also measured by thermo-couples during long-term operation for the BM. The highest point is at the pole edge and the temperature is $140 \mathrm{deg}-\mathrm{C}$. The measured temperatures almost agree with those from the analysis. Vibration measurement was carried out at the center of the end plate of the BM. A laser displacement gauge was used for two direction measurements. The measured results are $9 \mu \mathrm{m}(\mathrm{p}-\mathrm{p})$ and $12 \mu \mathrm{m}(\mathrm{p}-\mathrm{p})$ for vertical and longitudinal directions, respectively. This result shows that the amplitude is small enough for the end plate to support a beam duct. Based 
on these results, we have started mass-producing the BMs and QMs.

We employ a direct water cooling type MA cavity, and have been performing high power test at KEK in order to investigate optimum structure of a water vessel inside. The results were reflected to the design of the cavity for the RCS. For the RCS MA cavity, if we employ a cut core technique to get optimum $\mathrm{Q}$ value of 2 , the gap space of the core is less than $1 \mathrm{~mm}$. From the high power test results, we have found that the roughness on the cut surface was related to the heating on it. Some tests of more smooth cutting procedures such as grindstone cutting rather than water jet cutting are underway.

We have two types of alumina ceramics vacuum ducts[10]. One is $1.5 \mathrm{~m}$ long with a circular cross section for use in the QM. The other is $3.5 \mathrm{~m}$ long and bending 15 degrees with a race-track cross section for use in the BM. These ducts are manufactured by joining several duct segments of $0.5-0.8 \mathrm{~m}$ in length by brazing. They have copper stripes on the outside surface to reduce the duct impedance. Figure 6 shows the ceramics duct for a $\mathrm{QM}$ and a race-track shape segment. A TiN film is coated inside the surface to reduce secondary electron emission. Mass-production of these ceramic ducts is underway.

Some R\&Ds are conducted for the collimator system and injection/extraction devices. Details are shown in this conference[11,12,13].

The RCS building is accomplished in April, but the electric equipment and air conditioning system will require a couple of more months for completion. The machine installation will be started in August, 2005. The beam test for the RCS is planned in May, 2007.

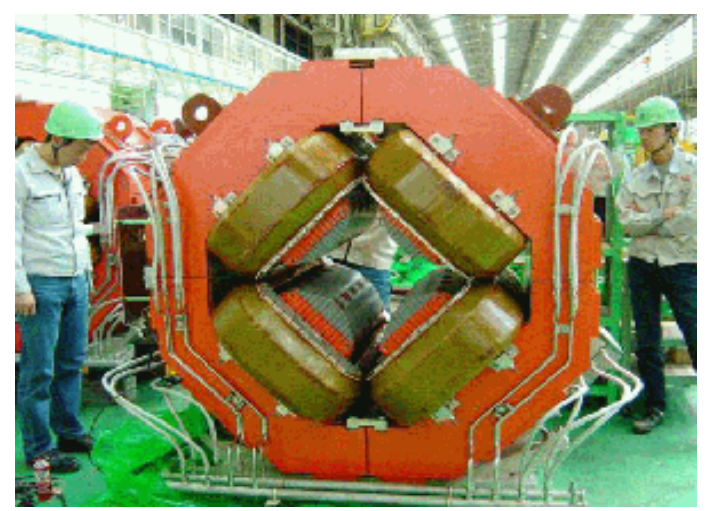

Figure 5: A large-bore quadrupole magnet for the 3-GeV RCS.

\section{0-GEV MR}

Most of the accelerator components were ordered except for the RF system and the injection and extraction systems. Mass production of 96 bending magnets and 216 quadupole magnets have been finished and mass field measurement is underway. In the mass measurements, 7 $\mathrm{m}$ long flip-flop coil for BMs and $2 \mathrm{~m}$ and $3 \mathrm{~m}$ harmonic
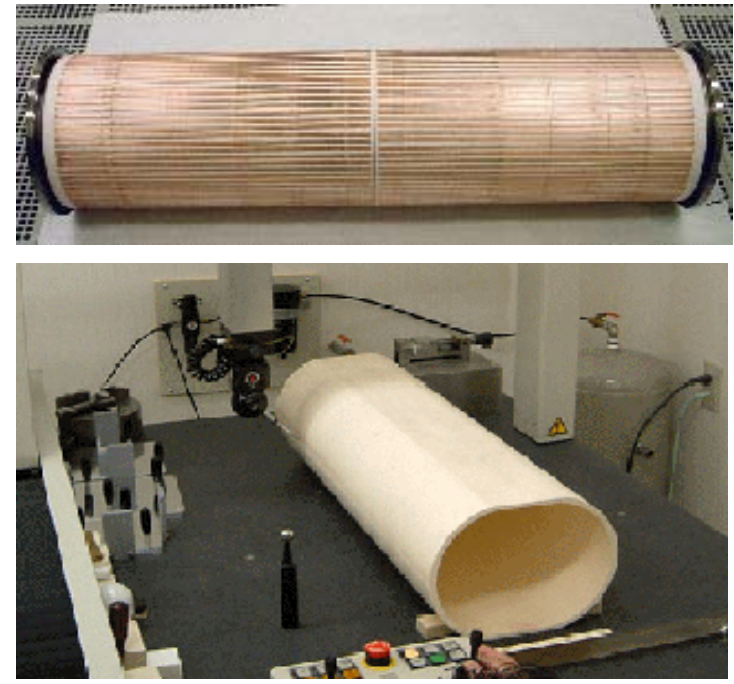

Figure 6: An alumina ceramics vacuum duct for a QM (top) and a race-track shape segment (bottom).

coil systems for QMs are constructed. Until now, mass measurement for $55 \mathrm{BMs}$ and $48 \mathrm{QMs}$ was carried out.

Opposite field septum magnet has been applied to the injection system[14]. The features of the system are a force-free structure, easy pulse excitation and the possibility of a large-aperture, thin-septum structure. This type of septum magnet was delivered and the test is underway.

All magnets were delivered and tested for the beam transport line from the RCS to the MR. We have a pulse bend for switching the 3-GeV beams from the MLF to the MR. Dummy load test of the power supply for the pulse bend was successfully carried out, its rise time and fall time are $33 \mathrm{msec}$ and $27 \mathrm{msec}$, respectively. These results satisfy the designed value of less than $40 \mathrm{msec}$. The pulse bend magnet will be tested by using this power supply.

To reduce the radiation dose, maintenance scenario is planned[15]. To reduce necessary time for the work, quick joints for vacuum flange, cooling water, and current lead are devised and tested. A mockup of the MR tunnel was constructed and set up the BM, QM, beam duct and others as shown in Fig. 7. By using this mockup,

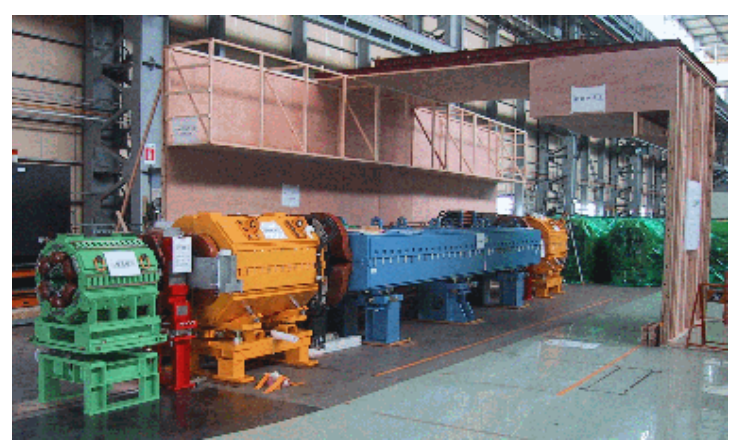

Figure 8: Mock up of the MR tunnel. Bending, Quadrupole and Sextupole magnets are in blue, orange and green, respectively. 
installation and maintenance procedures are carefully checked.

The civil engineering of the MR is divided into four sections, which will be constructed year by year. The installation of the accelerator components will be started on middle of 2005 and the beam commissioning is scheduled at the beginning of 2008 .

\section{EXPERIMENTAL FACILITIES}

Procurement and installation of large and heavy components, e.g., outer- and bottom-liners, a helium vessel, etc., have started in parallel to the MLF building construction[16]. Figure 8 shows the liner transportation work with a special carriage between the pine trees. R\&Ds are continued to investigate the pitting issue concerning the target lifetime limitation in collaboration with US/SNS team.

The installation to the switch yard for the Hadron Experimental Facilities will be started in March, 2006. Most of the magnets should be ready by that time. The KEK-Proton Synchrotron will be shut down at the end of 2005 and some magnets will be recycled. The construction will be completed and the first external beam from the MR will be expected in September, 2008.

At the Neutrino Facility, superconducting combined function magnets will be utilized and an R\&D program for the magnet is carried out[17]. The decay volume space was excavated and decay pipe with a length of 110 $\mathrm{m}$ was installed in this spring. Huge amount of concrete is pouring among the decay volume.

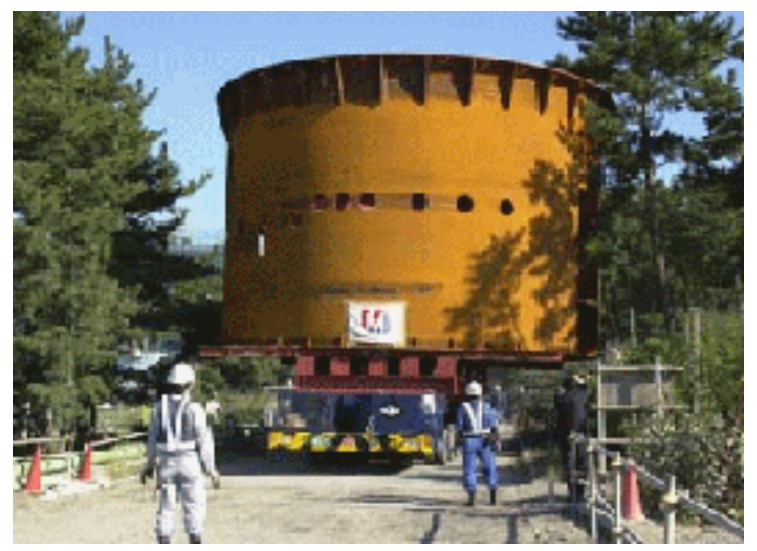

Figure 8: Transportation of the large outer liner along the road to the site of the MLF.

\section{SUMMARY}

The J-PARC accelerator, which consists of the linac, the RCS and the MR is based on many newly developed technologies. The developments have been successful, and commissioning of the J-PARC accelerator has been progressing well.
Installation of accelerator components has begun from the linac. The beam tests of the linac, the RCS and the MR are planned from September 2006, May 2007, and January 2008, respectively.

The beam power of the RCS at the initial stage is reduced to $0.6 \mathrm{MW}$, since the linac energy is reduced to $181 \mathrm{MeV}$. It is expecting that the linac energy upgrade to $400 \mathrm{MeV}$ will start immediately after or in parallel to the series of commissioning.

\section{ACKNOWLEDGEMENTS}

This paper is presented on behalf of all the J-PARC colleagues. The authors gratefully acknowledge their contributions.

\section{REFERENCES}

[1] "The Joint Project for High-Intensity Proton Accelerators", JAERI-Tech 99-056, KEK Report 99-4, and JHF-99-3 (1999).

[2] "Accelerator Technical Design Report for High-intensity Proton Accelerator Facility Project, J-PARC", JAERI-Tech 2003-044, KEK Report 2002-13 (2003).

[3] A. Ueno and Y. Yamazaki, Nucl. Instr. Meth. A300, p15 (1990).

[4] K. Yoshino et al., "Development of a DTL Quadrupole Magnet with a New Electroformed Hollow Coil for the JAERI/KEK Joint Project", XX International Linac Conference, Monterey, USA, pp.569-571, TUD10 (2000).

[5] S. Fu and T. Kato, "RF-chopper for the JHF proton linac", Nucl. Instr. Meth. A440, p296 (2000).

[6] T. Kato, KEK Report 92-10 (1992).

[7] “JHF Accelerator Design Study Report", KEK Report 9716 (1998).

[8] T. Kato et al., "Beam Study with RF Choppers in the MEBT of the J-PARC Proton Linac", the 2003 Particle Accelerator Conference, pp.1455-1457 (2003).

[9] Y. Kondo et al., "Beam Dynamics and Commissioning of the J-PARC Linac", Proc. of ICFA-HB2004, Bensheim / Darmstadt, Germany (2004).

[10] M. Kinsho et al., "Alumina Ceramics Vacuum Duct for the $3 \mathrm{GeV}-\mathrm{RCS}$ of the J-PARC", these proceedings, RPPE039.

[11] K. Yamamoto, "Development of the Collimator System for the $3 \mathrm{GeV}$ Rapid Cycling Synchrotron", these proceedings, WPAE013.

[12] T. Takayanagi et al., "Design of the Pulse Bending Magnets for the Injection System of the 3-GeV RCS in JPARC", these proceedings, MPPT006.

[13] J. Kamiya et al., "The Extraction Kicker System of the RCS in J-PARC", these proceedings, MPPT006.

[14] I. Sakai et al., "Opposite Field Septum Magnet System for the J-PARC 50-GeV Ring Injection", Proc. of EPAC2004, Lucerne, Switzerland, pp.1339-1341 (2004).

[15] M. Yoshioka et al., "Installation and Radiation Maintenance Scenario for J-PARC Main Ring", these proceedings, RPPE002.

[16] Y. Ikeda, "Status of J-PARC Project and JSNS Construction", ICANS-XVII, Santa Fe, NM, USA (2005).

[17] T. Nakamoto et al., "Development of Superconducting Combined Function Magnets for the Proton Transport Line for the J-PARC Neutrino Experiments", these proceedings, TOAA006. 